\title{
Meson-baryon threshold effects in the light-quark baryon spectrum
}

\author{
P. González \\ Departamento de Física Teórica e IFIC, \\ Universidad de Valencia - CSIC, E-46100 Burjassot, Valencia, Spain \\ J. Vijande \\ Departamento de Física Teórica e IFIC, \\ Universidad de Valencia - CSIC, E-46100 Burjassot, Valencia, Spain and \\ Departamento de Física Fundamental, \\ Universidad de Salamanca, E-37008 Salamanca, Spain
}

\author{
A. Valcarce \\ Departamento de Física Fundamental, \\ Universidad de Salamanca, E-37008 Salamanca, Spain
}

(Dated: November 1, 2018)

\begin{abstract}
We argue that selected $S$ wave meson-baryon channels may play a key role to match poor baryon mass predictions from quark models with data. The identification of these channels with effective inelastic channels in data analysis allows to derive a prescription which could improve the extraction and identification of baryon resonances.
\end{abstract}

PACS numbers: 14.20.-c,14.20.Gk 


\section{INTRODUCTION}

In the Particle Data Group (PDG) book [1] the light-quark ( $u$ and $d$ ) baryon spectrum is composed of forty resonances rated from one $(*)$ to four $(* * * *)$ stars. The PDG averagemass region below $1950 \mathrm{MeV}$ contains mostly four-star (well established) resonances, fourteen out of twenty three, the same being true for the $\Lambda$ strange sector, eight out of eleven. This makes this mass region the most suitable for testing any spectroscopic quark model. From the pioneering Isgur and Karl's non-relativistic quark model in the late 70's [2] more refined spectroscopic quark models for baryons, based on two-body interactions, have been developed [3, 4, 5, 6, 7, 8, 9]. We will refer to them as two-body quark models and we shall denote them generically as $3 q^{2 b}$. As an overall result the masses of the fourteen four-star resonances, most times with the exception of $N_{P_{11}}(1440)$ (see comments below), are rather well predicted $(\lesssim 100 \mathrm{MeV}$ difference with the PDG average value) by these models. Regarding the five three-star (likely to certain existence) resonances, the situation is much less favorable since the masses of two of them, $\Delta_{P_{33}}(1600)$ and $\Delta_{D_{35}}(1930)$, are generally overpredicted, up to $250 \mathrm{MeV}$ above the PDG average value. Let us note that a similar discrepancy is observed for $\Delta_{S_{31}}(1900)(* *)$ and $\Delta_{D_{33}}(1940)(*)(\gtrsim 100 \mathrm{MeV}$ difference with the PDG average value) which can be related to $\Delta_{D_{35}}(1930)$ as we shall show, and for $\Delta_{P_{31}}(1750)(*)$ (up to $200 \mathrm{MeV}$ above the PDG average). In the strange $\Lambda$ sector an outstanding overpredicted (by $80-150$ $\mathrm{MeV})$ state is the $\Lambda_{S_{01}}(1405)(* * * *)$. Henceforth we shall call anomalies these significantly overpredicted mass resonances.

In this article we carry out a general analysis of the anomalies: we identify them and we propose a plausible physical mechanism to give correctly account of their masses. To accomplish this task we shall first examine in detail in Sec. II the $3 q^{2 b}$ mass predictions and advance, through arguments of universality and consistency, the plausible role played by the coupling of three-quark components $(3 q)$ to relevant meson-baryon $(m B)$ channels. In Sec. III our qualitative considerations will be put on a more sound basis through a simplified model calculation. The successful description attained will drive us to prescribe in Sec. IV] the implementation of these relevant $m B$ channels in data analysis to improve the extraction of the anomalies. In Sec. $\mathrm{V}$ we revise alternative partial descriptions from existing quark models incorporating three-body interactions. Finally, in Sec. VI we summarize our main findings.

\section{TWO-BODY QUARK-MODEL PREDICTIONS AND MESON-BARYON THRESHOLD EFFECTS}

\section{A. Large-energy-step anomalies}

As explained next, most anomalies may be assigned either to a large radial energy excitation or to a $3 q^{2 b}$ configuration with large mass induced by quark Pauli blocking. We shall refer to them as large-energy-step anomalies.

1. Radial excitations: $\Delta_{P_{33}}(1600), N_{P_{11}}(1440)$

The $\Delta_{P_{33}}(1600)$ is the first positive parity excitation of $\Delta_{P_{33}}(1232) \equiv \Delta$. The large mass for $\Delta_{P_{33}}(1600)$ predicted by $3 q^{2 b}$ can be understood making use of an harmonic oscillator 
approximation, with $S U(6) \times O(3)$ symmetry $\left(S U(6) \supset S U(3)_{\text {Flavor }} \times S U(2)_{\text {Spin }}\right.$; for nonstrange quarks the flavor is specified by the isospin $I)$. Then the $\Delta_{P_{33}}(1600)$ may be assigned to the $\left(56, L^{P}=0^{+}\right)^{S=3 / 2}$ configuration in the $\mathcal{N}=2$ band (we shall obviate $I=3 / 2$ for $\Delta$ and $I=1 / 2$ when referring to nucleon). The band number $\mathcal{N}$ can be expressed as $\mathcal{N}=\left(2 n_{\rho}+\ell_{\rho}\right)+\left(2 n_{\lambda}+\ell_{\lambda}\right)$ where $\rho$ and $\lambda$ refer to the two Jacobi coordinates in a three-quark baryon and $\ell_{\rho}$ and $\ell_{\lambda}$ to the corresponding orbital angular momenta. The total orbital angular momentum of the system is given by $\vec{L}=\overrightarrow{\ell_{\rho}}+\overrightarrow{\ell_{\lambda}}$, and the parity $P$ by $P=(-)^{\ell_{\rho}+\ell_{\lambda}}=(-)^{\mathcal{N}}$. More specifically the $\Delta_{P_{33}}(1600)$ may be assigned to the first radial excitation of the $\Delta_{P_{33}}(1232):\left(n_{\rho}, n_{\lambda}\right)=(1,0)$ or $(0,1)$ and $\ell_{\rho}=0=\ell_{\lambda}$. From the harmonic oscillator energy, $E=(\mathcal{N}+3) \hbar \omega$ being $\omega$ the angular frequency, the first radial excitation, involving an $\mathcal{N}$ increase of two units, is higher in energy than the first orbital one, $\left(\ell_{\rho}, \ell_{\lambda}\right)=(1,0)$ or $(0,1)$ and $n_{\rho}=0=n_{\lambda}$, for which $\mathcal{N}$ increases only one unit. However this contradicts data since the $\Delta_{P_{33}}(1600)$ has lower mass than $\Delta_{D_{33}}(1700)$ or $\Delta_{S_{31}}(1620)$, the lowest negative parity excitations. This inversion problem, equivalent to the mass overprediction for $\Delta_{P_{33}}(1600)$, appears also for the Roper resonance, $N_{P_{11}}(1440)$, lower in mass than $N_{D_{13}}(1520)$ and $N_{S_{11}}$ (1535). Actually the solution of the Roper inversion has motivated many ad hoc quark model studies. Being our goal to get as much as possible a general understanding of the anomalies we shall include, in parallel to $\Delta_{P_{33}}(1600)$, the Roper resonance in our list.

Let us add that the conventional interpretation of $N_{P_{11}}(1440)$ and $\Delta_{P_{33}}(1600)$ as radial excitations we have assumed does not preclude other configuration assignments. In $N_{P_{11}}(1440)$ there is mixing with the orbital excitation $\left(70,0^{+}\right)_{\mathcal{N}=2}^{S=1 / 2}$ which could even be dynamically dominant. In $\Delta_{P_{33}}(1600)$ an alternative interpretation in terms of orbital excitations is also feasible as we shall show later on.

\section{Quark Pauli blocking induced states: $\Delta_{D_{35}}(1930), \Delta_{P_{31}}(1750)$}

Regarding the $\Delta_{D_{35}}(1930)$, say the lowest $\Delta\left(5 / 2^{-}\right)$energy state, it can be assigned to the $\left(56,1^{-}\right)^{S=3 / 2}$ configuration [10, 11]. Although the expression of $\mathcal{N}(\mathcal{N} \geq L)$ may suggest $\mathcal{N}=1$, this energy band is forbidden since being a completely symmetric state in isospin, $I=3 / 2$, and spin, $S=3 / 2$, the spatial part should also be completely symmetric whereas $\mathcal{N}=1$ only admits spatial states of mixed symmetry. Instead $\mathcal{N}=3$ according to parity with an $\mathcal{N}$ increase of two units, hence its predicted large mass. So quark Pauli blocking makes the system acquire two units of excitation, this time in the form $\left(\ell_{\rho}, \ell_{\lambda}\right)=(1,2)$ or $(2,1)$, instead of $\left(\ell_{\rho}, \ell_{\lambda}\right)=(1,0)$ or $(0,1)$.

An analogous situation occurs for $\Delta_{P_{31}}(1750)$, the lowest $\Delta\left(1 / 2^{+}\right)$PDG state. The configuration assigned, $\left(70,0^{+}\right)^{S=1 / 2}$, cannot combine with $\mathcal{N}=0$ which only admits completely symmetric spatial states, then $\mathcal{N}=2$ through two units of excitation $\left(\ell_{\rho}, \ell_{\lambda}\right)=(1,1)$, instead of $\left(\ell_{\rho}, \ell_{\lambda}\right)=(0,0)$.

\section{B. Meson-baryon threshold channels}

Given the large radial excitation energy and the large mass predicted for quark Pauli blocking induced states, one may wonder about the possibility that $4 q 1 \bar{q}$ components may be energetically competitive, despite the extra quark and antiquark masses. Thus, they could greatly contribute, altogether with $3 q$ components, to the formation of the bound 
structures $^{1}$. In order to examine this possibility at a phenomenological level we look for $4 q 1 \bar{q}$ components in the form of inelastic meson-baryon channels in relative $S$ wave (the lowest energy partial wave) with adequate quantum numbers to couple to the anomalies and with thresholds close above their PDG masses. We shall name these components mesonbaryon threshold channels or $m B$ channels.

\section{1. $\Delta_{D_{35}}(1930), \Delta_{D_{33}}(1940)$ and $\Delta_{S_{31}}(1900)$}

For $\Delta_{D_{35}}(1930)$ a simple inspection allows us to identify the following $m B$ channels: $\pi \Delta_{F_{35}}(1905), \omega \Delta$, and $\rho \Delta$ with thresholds at $2045 \mathrm{MeV}, 2014 \mathrm{MeV}$, and $2002 \mathrm{MeV}$, respectively (let us recall that $3 q^{2 b}$ mass predictions are $80-250 \mathrm{MeV}$ higher than the PDG average $1930 \mathrm{MeV}$ ). To discriminate among these channels we notice that going further with our argumentation we should expect the presence of $\Delta$ resonances close in mass to $\Delta_{D_{35}}(1930)$, whenever the same dominant configuration $\left(56,1^{-}\right)_{\mathcal{N}=3}^{S=3 / 2}$ and the same relevant thresholds are present. We shall refer to these resonances as partners. It turns out that $\Delta\left(1 / 2^{-}\right)$and $\Delta\left(3 / 2^{-}\right)$contain that configuration (in fact it is the only one common to these two deltas and $\Delta\left(5 / 2^{-}\right)$below $\left.2.2 \mathrm{GeV}\right)$. Moreover if we examine the PDG table we find the anomalies $\Delta_{S_{31}}(1900)$ and $\Delta_{D_{33}}(1940)$ sharing with $\Delta_{D_{35}}(1930)$ the $\omega \Delta$ and $\rho \Delta$ as $m B$ channels. This suggests $\Delta_{S_{31}}(1900)$ and $\Delta_{D_{33}}(1940)$ as partners of $\Delta_{D_{35}}(1930)$ and $\omega \Delta$ and/or $\rho \Delta$ as the possible relevant coupling to the binding of the three resonances.

Let us add for the sake of completeness that for $\Delta\left(1 / 2^{-}\right)$and $\Delta\left(3 / 2^{-}\right)$the $3 q^{2 b}$ first radial excitation $\left(70,1^{-}\right)_{\mathcal{N}=3}^{S=1 / 2}$, at about $2050 \pm 50 \mathrm{MeV}$, is not far above the average mass of their anomalies. However we shall justify later on the assignment of these radial excitations to $\Delta_{S_{31}}(2150)(*)$ and to a not yet extracted $\Delta_{D_{33}}$ resonance around the same energy.

\section{2. $\Delta_{P_{31}}(1750)$}

An analogous analysis based on the search of $m B$ channels can be carried out for $\Delta_{P_{31}}(1750)$ with a $60-200 \mathrm{MeV}$ mass overprediction from $3 q^{2 b}$. We find $\pi N_{S_{11}}(1650)$ and $\pi \Delta_{S_{31}}(1620)$ with thresholds at $1790 \mathrm{MeV}$ and $1760 \mathrm{MeV}$, respectively. Since both thresholds involve pions with $J^{P}=0^{-}$there cannot be $\Delta(J \neq 1 / 2)$ partners, with $\left(70,0^{+}\right)_{\mathcal{N}=2}^{S=1 / 2}$ and the same relevant thresholds. In consequence we have no further phenomenological indication on which threshold may be relevant.

\section{3. $\Delta_{P_{33}}(1600)$ and $N_{P_{11}}(1440)$}

For $\Delta_{P_{33}}(1600)$ with a $3 q^{2 b}$ mass overprediction of $80-250 \mathrm{MeV}$, the $\pi N_{D_{13}}(1520)$ channel (threshold at $1660 \mathrm{MeV}$ ) might contribute to the binding. Additionally $\sigma \Delta$ with a quite uncertain $S$ wave threshold due to the large interval accepted for the $\sigma$ mass $(400-1200$ $\mathrm{MeV}$ ) might play some role.

\footnotetext{
${ }^{1}$ Indeed the $\Delta_{D_{35}}(1930)$ was first interpreted as a hybrid state involving gluonic or nonvalence quark degrees of freedom [10] although this interpretation was questioned a few years later [12] through a revision of the role played by anharmonic perturbations.
} 
Analogously for $N_{P_{11}}(1440)$, with $3 q^{2 b}$ mass predictions ranging from $1410 \mathrm{MeV}$ to 1700 $\mathrm{MeV}$, the $\sigma N$ channel could play a relevant role. In fact, the explicit consideration of $\sigma N$ has allowed for a description of $N_{P_{11}}$ (1440) from a coupled meson-baryon channel calculation [13]. The $\pi N_{S_{11}}(1535)$ channel, although with threshold at $1675 \mathrm{MeV}$ quite above the PDG mass, could also have some effect. In both cases $\left(J_{\pi}=0=J_{\sigma}\right)$ there are no partners to be examined.

\section{Regular-energy-step anomalies}

Certainly meson-baryon channel coupling effects may be at work for other resonances not involving either large energy excitation steps or a large mass induced by quark Pauli blocking.

\section{1. $\Lambda_{S_{01}}(1405)$}

As a matter of fact the more generally accepted anomaly is the $\Lambda_{S_{01}}(1405)$ which has motivated a lot of studies being mostly interpreted, at the hadron level, as an $S$ wave $N \bar{K}$ quasi-bound system (in the chiral unitary approximation one of the poles couples mostly to $N \bar{K}[14]$ ). Alternatively, at the quark level, the identification of the lowest $3 q$ negative parity excitation of $\Lambda$ (predicted mass about $1550 \mathrm{MeV}$ ) with $\Lambda_{S_{01}}(1405)$ has been suggested, the difference in mass being attributed to the mass shift induced in $3 q\left(\Lambda\left(1 / 2^{-}\right)\right)$by its strong coupling to the $S$ wave $N \bar{K}$ channel (threshold at $1435 \mathrm{MeV}$ ) [4, 15]. Very recently a quantitative calculation along this line within a specific quark model framework has been performed [16]. Let us remark that although these explanations are formulated in terms of different degrees of freedom (hadrons or quarks) they may be somehow equivalent through the effectiveness of parameters, cutoffs...

Henceforth we shall assume that $\Lambda_{S_{01}}(1405)$ is a resonance induced by the coupling of $N \bar{K}$ to the lowest energy $3 q^{2 b}$ negative parity configuration with strangeness: $\left(70,1^{-}\right)_{\mathcal{N}=1}^{S=1 / 2}$ and flavor singlet, $I=0$. Since $J^{P}=0^{-}$for kaons no $\Lambda_{S_{01}}(1405)$ partners are expected. In fact the closest $\Lambda$ resonance, $\Lambda_{D_{03}}(1520)$, shares the same configuration but has no coupling to $N \bar{K}$.

$$
\text { 2. } \Delta_{F_{35}}(\sim 1720)
$$

For other light-quark resonances in the energy region under consideration $(\leq 1950 \mathrm{MeV})$ the inspection of data and $3 q^{2 b}$ mass predictions makes us conclude that mass overpredictions are not very significant with one possible exception. This corresponds to the lowest energy state of $\Delta\left(5 / 2^{+}\right)$. Since $L \neq 0$ the minimum possible $\mathcal{N}$ value is $\mathcal{N}=2$ according to parity. $3 q^{2 b}$ models predict two states in the $\mathcal{N}=2$ band with mass ranges $1870-1940 \mathrm{MeV}$ and $1930-2030 \mathrm{MeV}$ what seems to be in correspondence with the first and second PDG states $\Delta_{F_{35}}(1915)(* * * *)$ and $\Delta_{F_{35}}(2000)(* *)$. However the $\Delta_{F_{35}}(2000)$ is bizarre since its average mass is obtained from three different data analyses, two of them [17, 18] reporting a mass about $1720( \pm 60) \mathrm{MeV}$ and the other [19] giving a quite different value of $2200 \pm$ $125 \mathrm{MeV}$. Then by considering two differentiated resonances the $\Delta_{F_{35}}(\sim 1720)$ would be a clear candidate for an anomaly. Remarkably there is a $m B$ channel, the $\pi N_{D_{15}}(1675)$ with 
threshold at $1815 \mathrm{MeV}$, which could contribute to the binding of this resonance at such low energy. Note additionally that the $3 q^{2 b}$ configuration corresponding to $L=L_{\min }=1$ $(\Longrightarrow S=3 / 2$ ) and $\mathcal{N}=2$ is forbidden in this case due to its antisymmetric orbital character. This Pauli blocking does not imply though an increase in $\mathcal{N}$ but an orbital reordering of the quarks to an available $L=2, \mathcal{N}=2$ configuration, hence its regularenergy-step character.

Therefore we will tentatively identify the $\Delta_{F_{35}}(\sim 1720)$ as the lowest energy state of $\Delta\left(5 / 2^{+}\right)$and interpret it as another meson-baryon, $\pi N_{D_{15}}(1675)$, induced resonance. For its $3 q^{2 b}$ configuration assignment there are two options: i) $\left(70,2^{+}\right)_{\mathcal{N}=2}^{S=1 / 2}$ and ii) $\left(56,2^{+}\right)_{\mathcal{N}=2}^{S=3 / 2}$.

If we opt for i) this configuration is also present in $\Delta\left(3 / 2^{+}\right)$where it could couple to $\pi N_{D_{13}}(1700)$ with threshold at $1840 \mathrm{MeV}$. It turns out that $N_{D_{13}}(1700)$ is almost degenerate to $N_{D_{15}}(1675)$ sharing the same dominant configuration $\left(70,1^{-}\right)_{\mathcal{N}=1}^{S=3 / 2}$. Then we should expect a companion resonance of $\Delta_{F_{35}}(\sim 1720)$ in $\Delta\left(3 / 2^{+}\right)$at about the same energy. By revisiting the PDG book we find that $\Delta_{P_{33}}(1600)$ is assigned a mass around $1700 \mathrm{MeV}$ in Refs. [17, 18] in agreement with our expectation. Therefore our proposal of a distinctive $\Delta_{F_{35}}(\sim 1720)$ should be complemented with the consideration of the current $\Delta_{P_{33}}(1600)$ as a superposition of a resonance, companion of $\Delta_{F_{35}}(\sim 1720)$, and of the $3 q^{2 b}$ first radial excitation configuration which could also be affected by some $m B$ channel. Complementarily configuration ii) which is present in $\Delta\left(5 / 2^{+}\right), \Delta\left(3 / 2^{+}\right)$, and $\Delta\left(1 / 2^{+}\right)$, would be assigned to $\Delta_{F_{35}}(1915), \Delta_{P_{33}}(1920)$ and $\Delta_{P_{31}}(1910)$. The almost degenerate mass of these resonances seems to support this correspondence.

If instead we opt for ii) the same conclusion about the structure of $\Delta_{P_{33}}(1600)$ would be obtained since ii) also appears in $\Delta\left(3 / 2^{+}\right)$. Besides, as mentioned, ii) is also present in $\Delta\left(1 / 2^{+}\right)$where it could couple to $\pi N_{S_{11}}(1650)$ with threshold at $1790 \mathrm{MeV}$ (note that $N_{S_{11}}(1650)$ is almost degenerate and shares configuration with $N_{D_{15}}(1675)$ ). Then we should expect another companion resonance of $\Delta_{F_{35}}(\sim 1720)$ in $\Delta\left(1 / 2^{+}\right)$. We could identify this companion as the $\Delta_{P_{31}}(1750)$. In consequence we should conclude that the $3 q^{2 b}$ quark Pauli blocking induced configuration previously considered for $\Delta_{P_{31}}(1750)$, i.e., $\left(70,0^{+}\right)_{\mathcal{N}=2}^{S=1 / 2}$, should be instead assigned to the next $\Delta\left(1 / 2^{+}\right)$resonance $\Delta_{P_{31}}(1910)$ (note that in such a case no coupling to any relevant $m B$ channel would be needed for this configuration). Complementarily configuration i) would give account in this case of $\Delta_{F_{35}}(1915)$ and $\Delta_{P_{33}}(1920)$. Unfortunately being in any case a resonance induced through pions, $J^{P}=0^{-}$, there are no $\Delta$ partners which could help to decide in favor of one of the options.

To finish this section we represent in Fig. 1] the $3 q^{2 b}$ mass predictions based on Ref. [4] (the numerical values will be given in Table II) as compared to the experimental mass intervals for the anomalies.

\section{NAIVE MODEL CALCULATION}

To go beyond the qualitative analysis of the anomalies we have carried out some dynamical input is required. In the last years there has been an important progress in the development of dynamical coupled-channel (DCC) models of $\pi N$ scattering in the resonance region below $2 \mathrm{GeV}$ [20, 21, 22]. These models introduce bare baryon states to represent the quark core components of the resonances. Such components can be identified with constituent quark model predictions. The resonance, $R$ associated with a bare baryon state is induced by effective vertex interactions $R \rightarrow m B$ and $R \rightarrow \pi \pi N$. In practice the masses of the bare 
states are parameters of the model which are determined by fitting data (other parameters as effective coupling constants and form factors are fixed, as much as possible, within reasonable ranges). The most recent fit to $\pi N$ elastic scattering data (not including $\pi N \rightarrow \pi \pi N$ ) [21] indicates clearly that bare masses are higher than the PDG's resonance positions. This suggests these coupled-channel schemes as the appropriate frameworks for a thorough study of the anomalies. However this is a formidable task out of the scope of this article which has a rather exploratory character. Instead we shall perform a simplified quark model calculation along the lines followed in the meson case to evaluate $2 q 2 \bar{q}$ effects [23].

We shall consider a system of one confined channel, the $3 q^{2 b}$, in interaction with one free-channel, a meson-baryon threshold channel $m B$, with a hamiltonian matrix:

$$
[H] \simeq\left(\begin{array}{cc}
M_{m}+M_{B} & a \\
a^{*} & M_{3 q^{2 b}}
\end{array}\right)
$$

where $M_{3 q^{2 b}}$ stands for the mass of the $3 q^{2 b}$ state, $M_{m}$ and $M_{B}$ for the masses of the meson and baryon respectively and $a$ for a fitting parameter giving account of the interaction $(a$ could correspond for instance to a ${ }^{3} P_{0}$ transition hamiltonian matrix element). The effect of the interaction on the masses is easily obtained by diagonalization. The corresponding eigenvalues are

$$
M_{ \pm}=\left(\frac{M_{3 q^{2 b}}+\left(M_{m}+M_{B}\right)}{2}\right) \pm \sqrt{\left(\frac{M_{3 q^{2 b}}-\left(M_{m}+M_{B}\right)}{2}\right)^{2}+|a|^{2}}
$$

where $M_{-}$is smaller that $\left(M_{m}+M_{B}\right)$ and $M_{+}$is bigger than $M_{3 q^{2 b}}$.

It is noteworthy the correspondence between this simplified model and a truncated DCC model calculation. So $M_{3 q^{2 b}}$ represents a bare resonance mass, $M_{m}$ and $M_{B}$ meson and baryon masses which have been approximated by the experimental values, and $a$ a constant giving account of the bare resonance- $m B$ effective coupling.

In order to proceed to calculate the eigenvalues we have to choose a particular $3 q^{2 b}$ model and establish a criterion for the choice of the $m B$ channel for each anomaly. We shall use as $M_{3 q^{2 b}}$ the values calculated in Ref. [4]. Since only the energy band and not the detailed configuration corresponding to each value has been published an educated guess has been done. As $m B$ we shall take for granted the $N \bar{K}$ channel for $\Lambda_{S_{01}}(1405)$. For $\Delta_{D_{35}}(1930)$, $\Delta_{D_{33}}(1940)$ and $\Delta_{S_{31}}(1900)$ we shall select $\rho \Delta$ (equivalently we could have preferred the almost degenerate $\omega \Delta$ ) as suggested by our phenomenological analysis. For the same reason $\pi N_{D_{15}}(1675)$ will be employed for $\Delta_{F_{35}}(\sim 1720)$. For $\Delta_{P_{31}}(1750)$ we shall use $\pi N_{S_{11}}(1650)$ since this coupling is favored at least in one of the two possible configuration assignments previously discussed.

Regarding $N_{P_{11}}(1440)$ and $\Delta_{P_{33}}(1600)$ the situation is rather intricate due to the alternative interpretations (radial and/or orbital excitations) available. From the particular $3 q^{2 b}$ model we use, the value $M_{3 q^{2 b}}=1540 \mathrm{MeV}$ corresponding to a dominant first radial excitation of $N$, can be unambiguously assigned to $N_{P_{11}}(1440)$. Then if we use the nominal PDG average value for the $\sigma$ mass $(600 \mathrm{MeV})$ the $\sigma N$ threshold is about the same energy than $M_{3 q^{2 b}}$ and closer to the PDG mass of the Roper than $\pi N_{S_{11}}(1535)$ with threshold at 1675 $\mathrm{MeV}$. This suggests the selection of the energetically more competitive $\sigma N$ channel as the possible relevant one. We should keep in mind though that the selection could be different favoring $\pi N_{S_{11}}$ (1535) for other choices of the $\sigma$ mass and the $3 q^{2 b}$ model. Actually given the large mass and width of the $\sigma$ it is also possible that both $m B$ channels may be contributing 


\begin{tabular}{|c|c|}
\hline PDG average & Meson-baryon channels \\
\hline$\Delta_{P_{33}}(1600)(* * *)$ & $\pi N_{P_{13}}(1520)$ \\
\hline$N_{P_{11}}(1440)(* * * *)$ & $\sigma N$ \\
\hline \hline$\Delta_{D_{35}}(1930)(* * *)$ & \\
$\Delta_{D_{33}}(1940)(*)$ & $\rho$ \\
$\Delta_{S_{31}}(1900)(*)$ & \\
\hline$\Delta_{P_{31}}(1750)(*)$ & $\pi N_{S_{11}}(1650)$ \\
\hline \hline$\Delta_{F_{35}}(\approx 1720)$ (N.C. $)$ & $\pi N_{D_{15}}(1675)$ \\
\hline
\end{tabular}

TABLE I: Light-quarks PDG resonances from Ref. 1] (N.C. means non-cataloged) representing spectroscopic anomalies and corresponding selected meson-baryon threshold channels.

to the binding. Consequently our selection should be considered as an effective one getting an insight into the relevant meson-baryon threshold effects.

Concerning the $\Delta_{P_{33}}(1600)$ it could be just the companion of $\Delta_{F_{35}}(\sim 1720)$ with the $3 q^{2 b}$ first radial excitation of $\Delta$ being hidden in its large width. However, although with different values of $I$ and $S$, the first radial excitations of $\Delta$ and $N$ share the same $S U(6) \times$ $O(3)$ configuration. On the other hand the $B$ components of the respective $m B$ channels $\left(N_{D_{13}}(1520)\right.$ vs. $\pi N_{S_{11}}(1535)$ and $\Delta$ vs. $\left.N\right)$ also share $S U(6) \times O(3)$ configuration. Then it seems natural to assume that meson-baryon threshold effects may be also acting on the first radial excitation of the $\Delta$. Moreover the low PDG average mass of $\Delta_{P_{33}}(1600)$ as compared to $1720 \mathrm{MeV}$, the approximated mass of the companion resonance mentioned above, seems to reinforce this idea. By using again the PDG nominal $\sigma$ mass we realize that only the $\pi N_{D_{13}}(1520)$ channel has now a threshold (at $1660 \mathrm{MeV}$ ) below the $3 q^{2 b}$ first radial excitation at $1790 \mathrm{MeV}$. This suggests the selection of $\pi N_{D_{13}}(1520)$ as the relevant $m B$ channel (the same caution and comments as in the Roper case should be applied here).

In Table \ we list all the light-quark baryon anomalies and the $m B$ channels plausibly contributing to their bindings according to our discussion (for $\Delta_{P_{33}}(1600)$ we list only the one corresponding to the radial excitation; for $\Delta_{P_{31}}(1750)$ the listed $m B$ channel refers only to one of the options commented above). In Fig. 2 we represent the selected energy thresholds, $M_{m}+M_{B}$ (values in Table 【I) as compared to the experimental mass interval for the anomalies.

\section{A. $\quad M_{-}$resonances}

Although the value of $|a|$ might vary depending on the configurations involved in each $(m B)-3 q$ coupling we shall use for the sake of simplicity the same value in all cases. The $M_{-}$results for $|a|=85 \mathrm{MeV}$ are numerically detailed in Table I where the values for $M_{3 q^{2 b}}$

and for $\left(M_{m}+M_{B}\right)$ in the chosen $m B$ channel as well as their probabilities to give $M_{-}$are also displayed. As can be checked the improvement of the description with respect to $3 q^{2 b}$ is astonishing. All the predicted $M_{-}$masses lye very close to the PDG average masses for the anomalies. In Fig. 3 the $M_{-}$values for $|a|=85 \mathrm{MeV}$ are drawn as compared to the experimental mass intervals.

We interpret these results as providing strong quantitative support to our former qualitative description of the anomalies. Regarding their nature a look at the probabilities reveal 
they are mostly meson-baryon states. Actually a meson-baryon probability greater or equal than $50 \%$ can serve as a criterion to identify an anomaly. Nonetheless the coupling to the $3 q$ component is essential to lower their masses making them more stable against decay into $m+B$.

Furthermore the resulting probabilities could be used, at least in the cases where $M_{m}+M_{B}$ is very close above the PDG average mass, to make a quantitative estimation of the effective coupling constant of the physical state to states of the continuum [24].

It should be emphasized that similar results could be obtained for any other spectroscopic $3 q^{2 b}$ model through a fine tuning of the value of $|a|$ (note that the small value of $|a|$ as compared to $M_{3 q^{2 b}}$ and $\left(M_{m}+M_{B}\right)$ provides an a posteriori validation of our method). This comes from the expression of the eigenvalues where it is clear that even for $|a|=0$ one gets $M_{-}=M_{m}+M_{B}$ which according to our $m B$ choice is much closer to the PDG mass of the anomaly than $M_{3 q^{2 b}}$, see Fig. 2. This means that concerning the mass of the anomalies the coupling of meson-baryon to $3 q$ components may play the role of a general healing mechanism for spectroscopic models.

\section{B. $M_{+}$resonances}

The values of $M_{+}$, the second solutions, as well as the next $3 q^{2 b}$ mass predictions taken from Ref. [4] are shown in Table III as compared to the PDG average masses and widths of the anomalies and to the mass of the next PDG equally labeled resonances. The $M_{+}$states, with masses above $M_{3 q^{2 b}}$, are dominantly $3 q$ components (with complementary $(m B)-3 q$ probabilities with respect to $M_{-}$) and may decay into $m+B$. A look at Table III shows that for $\Delta_{F_{35}}(\sim 1720)$ and $\Lambda_{S_{01}}(1405)$ the $M_{+}$state can be clearly assigned altogether with the next $3 q^{2 b}$ state to the next PDG resonances $\Delta_{F_{35}}(1905)$ and $\Lambda_{S_{01}}(1670)$ respectively. In the first case both $M_{+}$and the next $3 q^{2 b}$ mass are above the next PDG mass whilst in the second case both lye below it. This is in accord with the quite general quark model tendency of over (under) prediction for states in the $\mathcal{N}=2$ (1) bands.

The same type of assignment can be done for $\Delta_{S_{31}}(1900)$ where the $M_{+}$and the next $3 q^{2 b}$ masses lye respectively close above and below the next PDG mass average $\Delta_{S_{31}}(2150)$.

\begin{tabular}{|c|rr|rr|r|r|}
\hline PDG Resonance & $m B$ threshold & Prob. & $3 q^{2 b}$ & Prob. & $M_{-}$ & Experiment \\
\hline \hline$\Delta_{P_{33}}(1600)(* * *)$ & {$\left[\pi N_{P_{13}}(1520)\right](1660)$} & $81.1 \%$ & 1795 & $18.9 \%$ & 1619 & $1550-1700$ \\
\hline$N_{P_{11}}(1440)(* * * *)$ & {$[\sigma N](1540)$} & $50.0 \%$ & 1540 & $50.0 \%$ & 1455 & $1420-1470$ \\
\hline$\Delta_{D_{35}}(1930)(* * *)$ & & $83.4 \%$ & 2155 & $16.6 \%$ & 1964 & $1900-2020$ \\
$\Delta_{D_{33}}(1940)(*)$ & {$[\rho \Delta](2002)$} & $82.2 \%$ & 2145 & $17.8 \%$ & 1962 & $1840-2040^{\dagger}$ \\
$\Delta_{S_{31}}(1900)(*)$ & & $81.5 \%$ & $214018.5 \%$ & 1961 & $1850-1950$ \\
\hline$\Delta_{P_{31}}(1750)(*)$ & {$\left[\pi N_{S_{11}}(1650)\right](1790)$} & $62.8 \%$ & $183537.2 \%$ & 1725 & $1710-1780$ \\
\hline$\Delta_{F_{35}}(\approx 1720)($ N.C. $)$ & {$\left[\pi N_{D_{15}}(1675)\right](1815)$} & $74.4 \%$ & $191025.6 \%$ & 1765 & $1660-1785^{\dagger \dagger}$ \\
\hline$\Lambda_{S_{01}}(1405)(* * * *)$ & {$[\bar{K} N](1434)$} & $78.2 \%$ & $155021.8 \%$ & 1389 & $1400-1410$ \\
\hline
\end{tabular}

TABLE II: Predicted masses, $M_{-}$, for the anomalies as compared to experimental data from Ref. [1], Ref. [19] (indicated by the superindex $\dagger$ ), and Ref. [18] (indicated by the superindex $\dagger \dagger$ ). Two-body quark-model masses $\left(3 q^{2 b}\right)$ are taken from Ref. [4]. Probabilities (Prob.) for meson-baryon and $3 q$ components are also shown. All masses are in $\mathrm{MeV}$. 


\begin{tabular}{|c|c|c|c|c|}
\hline PDG anomaly & $\Gamma(\mathrm{MeV})$ & $M_{+}$ & Next $3 q^{2 b}$ & Next PDG resonance \\
\hline$N_{P_{11}}(1440)(* * * *)$ & $\sim 300$ & 1625 & 1770 & $N_{P_{11}}(1710)(* * *)$ \\
\hline$\Delta_{P_{33}}(1600)(* * *)$ & $\sim 350$ & 1836 & 1915 & $\Delta_{P_{33}}(1920)(* * *)$ \\
\hline$\Delta_{D_{35}}(1930)(* * *)$ & $\sim 360$ & 2193 & 2165 & $\Delta_{P_{35}}(2350)(*)$ \\
$\Delta_{D_{33}}(1940)(*)$ & $\sim 200$ & 2185 & 2080 & \\
$\Delta_{S_{31}}(1900)(*)$ & $\sim 200$ & 2180 & 2035 & $\Delta_{S_{31}}(2150)(*)$ \\
\hline$\Delta_{P_{31}}(1750)(*)$ & $\sim 300$ & 1900 & 1875 & $\Delta_{P_{31}}(1910)(* * * *)$ \\
\hline$\Delta_{F_{35}}(\approx 1720)($ N.C. $)$ & $\sim 140$ & 1960 & 1990 & $\Delta_{F_{35}}(1905)(* * * *)$ \\
\hline$\Lambda_{S_{01}}(1405)(* * * *)$ & 50 & 1595 & 1615 & $\Lambda_{S_{01}}(1670)(* * * *)$ \\
\hline
\end{tabular}

TABLE III: Predicted masses, $M_{+}$, as compared to the masses of the next $3 q^{2 b}$ states from Ref. [4] and the next PDG resonances from Ref. [1]. The widths of the corresponding anomalies are also shown. All masses are in $\mathrm{MeV}$.

For the sake of consistency we should also expect $\Delta_{D_{35}}$ and $\Delta_{D_{33}}$ resonances around 2150 $\mathrm{MeV}$ which have not been reported. This may have to do with the bigger proliferation of $3 q^{2 b}$ states about that energy (three for $\Delta(5 / 2-)$ and four for $\Delta(3 / 2-)$ against two for $\Delta(1 / 2-))$ what may make difficult its experimental disentanglement. Indeed the large width of $\Delta_{D_{35}}(1930)$ may be also including the effect of the missed resonance. In any case there is need of further data analysis to clarify the situation.

For $\Delta_{P_{31}}(1750)$ the $M_{+}$state could also play some role in its large width although an assignment to $\Delta_{P_{31}}(1910)$ seems the most logical.

Finally for $N_{P_{11}}(1440)$ and $\Delta_{P_{33}}(1600)$ the $M_{+}$states with masses $85 \mathrm{MeV}$ below $N_{P_{11}}(1710)$ and $\Delta_{P_{33}}(1920)$ respectively may be influencing the large widths of the anomalies as well as the widths of these next PDG states.

\section{Other thresholds}

Let us note that the expression given above for the eigenvalues is symmetric under the exchange of $M_{3 q^{2 b}}$ and $\left(M_{m}+M_{B}\right)$. Then mass corrections to $3 q^{2 b}$ states could alternatively come from meson-baryon channels above the $3 q^{2 b}$ mass predictions. However for an anomaly with $3 q^{2 b}$ mass prediction far above the PDG average we do not expect these contributions to be physically relevant in the sense of having any effect on its mass. Actually, in our simplified treatment a much larger value of $|a|$ would be required to get a correct mass shift from these thresholds, putting into question the very validity of the model. On the other hand, $m B$ channels different than the selected ones could have dynamically some effects through higher partial waves. In the spirit of quark model calculations we consider the $m B$ channels we have selected (which may not have a precise experimental correspondence) as effective ones giving account of the couplings of $3 q^{2 b}$ states with meson-baryon components.

\section{RESONANCE EXTRACTION PRESCRIPTION (REP)}

Most light-quark baryon resonances are extracted from data through a parametrization of $\pi N$ scattering partial waves. This usually refers to a multichannel scattering matrix including effective inelastic channels. The consideration of multichannel couplings becomes 
relevant when an important channel opens within the width of a resonance. Let us emphasize that this is so even if the threshold of the channel is above the mass of the resonance. Actually the consideration of the $\sigma N$ (also named $\epsilon N$ ) channel as an effective inelastic channel in some data analyses becomes relevant for the experimental extraction of the Roper resonance $N_{P_{11}}(1440)$, see for instance reference [17]. In parallel the extraction of $\Delta_{D_{35}}(1930)$ as a distinctive resonance is associated in some data analysis to the explicit inclusion of a $\rho \Delta$ effective inelastic channel [17, 19]. Hence a certain correspondence between efficient inelastic channels in data analyses and our selected meson-baryon threshold channels considered for the anomalies shows up for $\Delta_{D_{35}}(1930)$ and $N_{P_{11}}(1440)$. This suggests the generalization of this correspondence. Therefore we propose the explicit inclusion in data analyses of the selected meson-baryon threshold channels in order to make easier the extraction of the anomalies. In this way an improvement (star-number increase) over the current PDG star-status could result for all of them as well as consistency among different analysis of approximately the same set of data might be attained. We shall call REP (Resonance Extraction Prescription) this proposal.

\section{ALTERNATIVE EXPLANATIONS: THREE-BODY QUARK-MODEL PRE- DICTIONS}

Our description of the anomalies does not exclude in principle other possible equivalent effective treatments. As already mentioned alternative explanations based on poles in a meson-baryon coupled channel approach can be also found in the literature for $N_{P_{11}}(1440)$ and $\Lambda_{S_{01}}(1405)$. In this last case even a meson-baryon bound state interpretation is feasible. From the point of view of quark approaches it is worthwhile to comment that for lightquark baryons there exist at least two quark model calculations in the literature beyond $3 q^{2 b}$, giving a proper account of the masses of the large-energy-step anomalies. One of these models [25] incorporates a two-sigma exchange potential apart from a one-gluon exchange and confinement interactions. The other is a collective model where baryons appear as vibrations and rotations of a three-quark $Y$-shaped string-like configuration [26]. The energy systematic of these models is such that the energy step associated to a radial excitation or a quark Pauli blocking induced configuration gets reduced to approximately half of its $3 q^{2 b}$ value in agreement with data. On the contrary the predictions for regular-energy-step states do not vary significantly from $3 q^{2 b}$. This explains why the $\Lambda_{S_{01}}(1405)$ is out of the systematic (Ref. [25] predicts a mass of $1550 \mathrm{MeV}$ and Ref. [26] $1640 \mathrm{MeV}$ ) as well as it would be the $\Delta_{F_{35}}(\sim 1720)(1830 \mathrm{MeV}$ in [25] and $1921 \mathrm{MeV}$ in [26]) in case of its confirmation as a distinctive resonance.

Let us notice though that these exceptions could be put in their right masses by coupling them to the relevant meson-baryon threshold channels. In this manner a description of similar quality to the one in Sec. III could be reached. Note that the correct large-energy-step model predictions would not require now relevant meson-baryon threshold channels. Therefore an alternative spectral description where the large-energy-step anomalies correspond to $3 q$ states is feasible. 


\section{SUMMARY AND FINAL COMMENTS}

To summarize we propose that $4 q 1 \bar{q}$ components, in the form of $S$ wave meson-baryon channels which we identify, play an essential role in the description of the anomalies, say baryon resonances very significantly overpredicted by three-quark models based on two-body interactions. As a matter of fact by considering a simplified description of the anomalies as systems composed of a free meson-baryon channel interacting with a three-quark confined component we have shown they could correspond mostly to meson-baryon states but with a non-negligible $3 q$ state probability which makes their masses to be below the meson-baryon threshold. The remarkable agreement of our results with data in all cases takes us to refine our definition and propose the dominance of meson-baryon components as the signature of an anomaly. Relying on the $3 q^{2 b}$ mass predictions from Ref. [4] the Roper resonance, $N_{P_{11}}(1440)$, might be just in the limit being a quasianomalous state with a $50 \%$ probability of $\sigma N$. For the other identified anomalies, $\Delta_{P_{33}}(1600), \Delta_{D_{35}}(1930), \Delta_{D_{33}}(1940), \Delta_{S_{31}}(1900)$, $\Delta_{P_{31}}(1750), \Lambda_{S_{01}}(1405)$ and the non-cataloged $\Delta_{F_{35}}(\sim 1720)$, the meson-baryon component probability is magnified.

Though it is probable that these results may vary quantitatively when a more complete dynamical coupled-channel calculation is carried out we think it is reasonable not to expect major qualitative changes. Then it is plausible, given their dominant meson-baryon character, that the Roper resonance and specially the other Magnificent Seven anomalies be dynamically generated via simplified effective meson-baryon and/or meson-meson-baryon coupled channel calculations involving only a selected number of channels and couplings. Indeed this has been shown for $N_{P_{11}}(1440)$ and $\Lambda_{S_{01}}$ (1405), the effectiveness of the parameters possibly taking implicitly into account the nonconsidered three-quark components. In particular the effective dynamical generation of $\Delta_{F_{35}}(\sim 1720)$ could be interpreted as given a strong support to our proposal of considering it a distinctive resonance. Effective meson-baryon coupled channel studies would be also welcome to clarify the situation for other anomalies where alternative three-quark descriptions are available. The information obtained in this manner could be complemented with the one coming from quark model evaluations of hadronic transition processes in order to shed some light on the very detailed nature of the anomalies. With respect to this let us remind that the "three-body quark" and the "two-body quark + meson-baryon" wave functions may be rather different.

More complete studies are also needed to extract some conclusion about the possible anomalous character of some other resonances, apart from the Roper, in the nucleon sector. Our easy identification of most $\Delta$ anomalies may have to do with the quite small mixing present in their assigned anomalous configurations. Actually mixing with nonanomalous ones might play a role for some nucleon excitations making them not to show up as very significantly overpredicted mass states. Particularly states in the $\mathcal{N}=2$ band: $N_{P_{11}}(1710)(* * *), N_{P_{13}}(1720)(* * * *), N_{F_{15}}(1680)(* * * *)$, would deserve attention.

To finish we should comment on the possible drawbacks of our approach. Our description, based on a phenomenological analysis and on a healing formula for mass corrections to quark models predictions, relies on the assumption of a significant coupling between specific $3 q$ states and relevant meson-baryon channels. No physical mechanism underlying these particular couplings is detailed. Indeed, our effective treatment might correspond to different physical mechanisms depending on the anomaly. Note that for $\Lambda_{S_{01}}(1405)$, at difference with $\Delta$ cases, a diquark dominant induced coupling seems to be favored. Moreover, the consideration that our effective meson-baryon threshold channel might be either replacing the 
influence of other couplings (including other meson-baryon partial waves) or even correcting three-quark models dynamical deficiencies may be too naive. Besides we should keep in mind that except for $\Delta_{P_{33}}(1600)$ and $\Delta_{D_{35}}(1930)$ the existence of all the magnificent anomalies is fair or poorly established rating $(* *)$ or $(*)$ in the PDG book or even non-cataloged $\left(\Delta_{F_{35}}(\sim 1720)\right)$. Actually as established by the PDG editors most high lying states are questionable. Hence the possibility that some of them do not remain in time is opened.

Keeping in mind these caveats the universality, consistency and simplicity of our description make us confident that the implemented physical ingredients will remain essential in further theoretical evaluations. On the other hand from the experimental point of view the application of our Resonance Extraction Proposal (REP) of implementing selected mesonbaryon threshold channels in data analyses might add certainty to the existence of some resonances and at the same time help to reconcile competing and sometimes not very compatible partial wave analyses. Future work along these lines would be encouraging.

\section{ACKNOWLEDGMENTS}

This work has been partially funded by the Spanish Ministerio de Educación y Ciencia and EU FEDER under Contract No. FPA2007-65748, by European Integrated Infrastructure Initiative 506078, by Junta de Castilla y León under Contract No. SA016A17, and by the Spanish Consolider-Ingenio 2010 Program CPAN (CSD2007-00042).

[1] W.-M. Yao et al., J. Phys. G 33, 1 (2006).

[2] N. Isgur and G. Karl, Phys. Lett. 72B, 109 (1977); Phys. Rev. D 19, 2653 (1979).

[3] B. Silvestre-Brac and C. Gignoux, Phys. Rev. D 32, 743 (1985).

[4] S. Capstick and N. Isgur, Phys. Rev. D 34, 2809 (1986).

[5] R. Sartor and Fl. Stancu, Phys. Rev. D 31, 128 (1985); Fl. Stancu and P. Stassart, Phys. Lett. B 269, 243 (1991).

[6] Y.-B. Dong, J.-C. Su, and S.-S. Wu, J. Phys. G 20, 73 (1994).

[7] L.Ya. Glozman and D.O. Riska, Phys. Rep. 268, 263 (1996); L.Ya. Glozman, W. Plessas, K. Varga, and R.F. Wagenbrunn, Phys. Rev. D 58, 094030 (1998).

[8] S. Capstick and W. Roberts, Prog. Part. Nucl. Phys. 45, S241 (2000) and references therein.

[9] A. Valcarce, F. Fernández, P. González, and V. Vento, Phys. Lett. B 367, 35 (1996); A. Valcarce, H. Garcilazo, and J. Vijande, Phys. Rev. C 72, 025206 (2005).

[10] R.E. Cutkosky, R.E. Hendrick, and R.L. Kelly, Phys. Rev. Lett. 37, 645 (1976).

[11] R.H. Dalitz, R.R. Horgan, and L.J. Reinders, J. Phys. G 3, L195 (1977).

[12] K.C. Bowler, P.J. Corvi, A.J.G. Hey, and P.D. Jarvis, Phys. Rev. Lett. 45, 97 (1980); P.J. Corvi, Phys. Lett. B 101, 349 (1981).

[13] O. Krehl, C. Hanhart, S. Krewald, and J. Speth, Phys. Rev. C 62, 025207 (2000); C. Schütz, J. Haidenbauer, J. Speth, and J.W. Durso, Phys. Rev. C 57, 1464 (1998).

[14] A. Müller-Groeling, K. Holinde, and J. Speth, Nucl. Phys. A 513, 557 (1990); D. Jido, J.A. Oller, E. Oset, A. Ramos, and U.G. Meissner, Nucl. Phys. A 755, 669 (2005); and references therein.

[15] S. Pakvasa and S.F. Tuan, Phys. Lett. B 459, 301 (1999) and references therein.

[16] S. Takeuchi and K. Shimizu, Phys. Rev. C 76, 035204 (2007). 
[17] D.M. Manley and E.M. Saleski, Phys. Rev. D 45, 4002 (1992).

[18] T.P. Vrana, S.A. Dytman, and T.-S.H. Lee, Phys. Rep. 328, 181 (2000).

[19] R.E. Cutkosky, C.P. Forsyth, J.B. Babcock, R.L. Kelly, and R.E. Hendrick, Proceedings of the IV International Conference on Baryon Resonances (Baryon 1980), edited by N. Isgur, Toronto 1980.

[20] A. Matsuyama, T. Sato, and T.-S.H. Lee, Phys. Rept. 439, 193 (2007) and references therein.

[21] B. Juliá-Díaz, T.-S.H. Lee, A. Matsuyama, and T. Sato, Phys. Rev. C 76, 065201 (2007).

[22] A.M. Gasparyan, J. Haidenbauer, C. Hanhart, and J. Speth, Phys. Rev. C 68, 045207 (2003) and references therein.

[23] T. Browder, S. Pakvasa, and A. Petrov, Phys. Lett. B 578, 365 (2004).

[24] C. Hanhart, arXiv:0711.0578 [hep-ph].

[25] B. Desplanques, C. Gignoux, B. Silvestre-Brac, P. González, J. Navarro, and S. Noguera, Z. Phys. A 343, 331 (1992).

[26] R. Bijker, F. Iachello, and A. Leviatan, Ann. Phys. 236, 69 (1994). 
FIG. 1: Mass predictions for the anomalies from Ref. [4] (dashed lines) as compared to the experimental mass intervals detailed in Table $\amalg$ (boxes). N.C. means non-cataloged resonance.

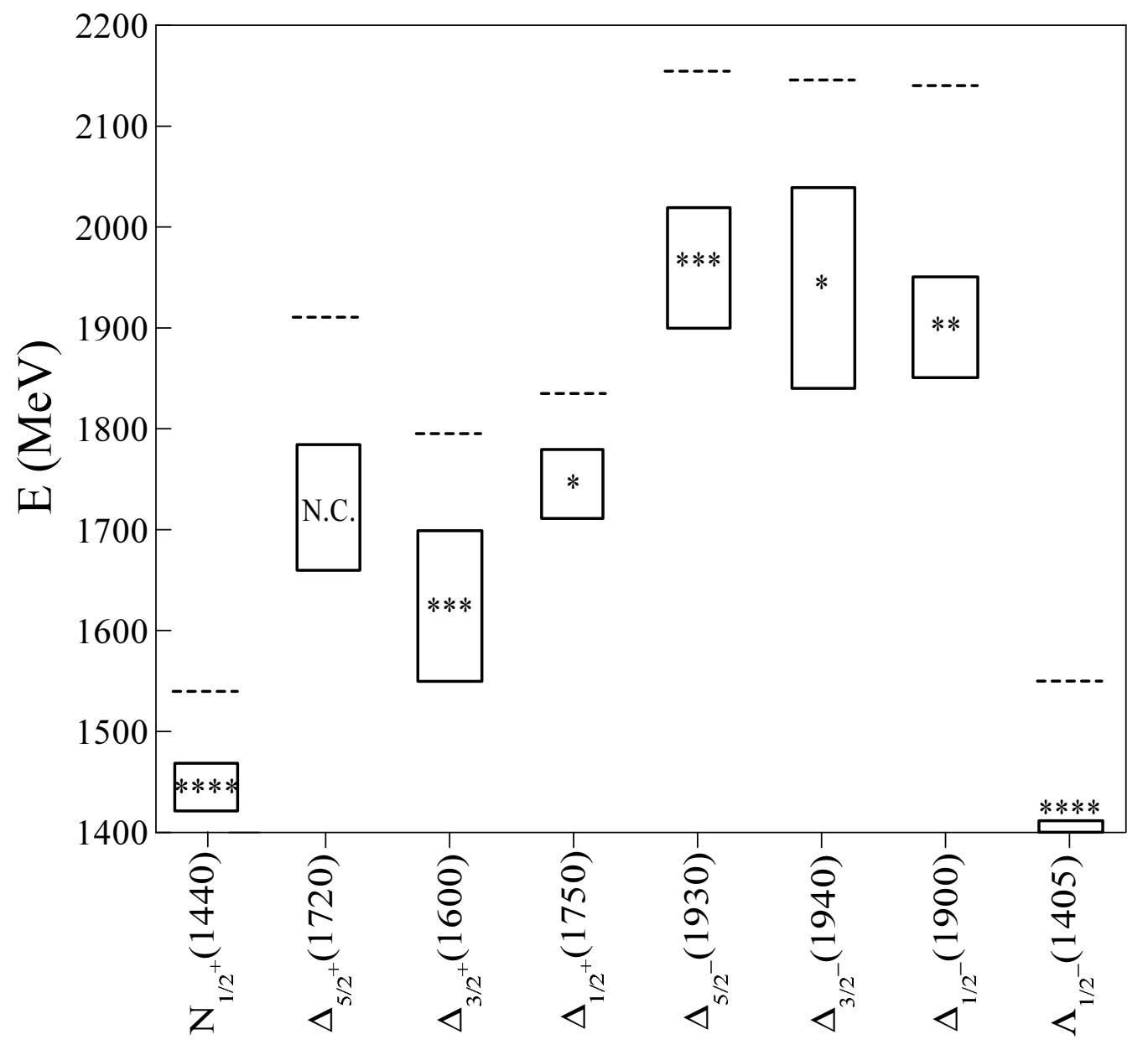

Figure 1 
FIG. 2: Selected energy thresholds (solid lines) as compared to the experimental mass intervals for the anomalies detailed in Table I (boxes). N.C. means non-cataloged resonance.

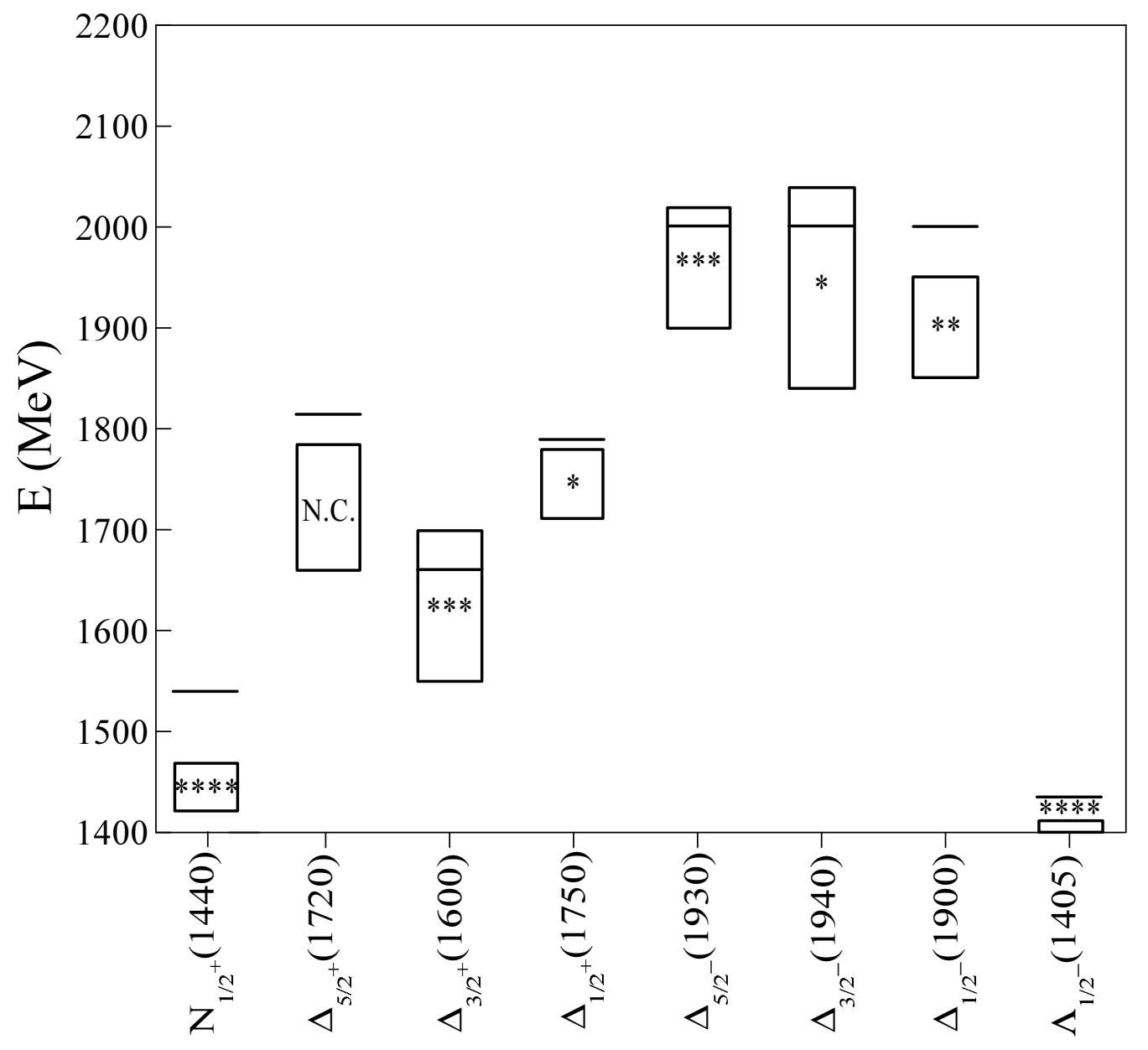

Figure 2 
FIG. 3: Predicted masses for the anomalies (dashed lines) as compared to the experimental mass intervals detailed in Table (boxes). N.C. means non-cataloged resonance.

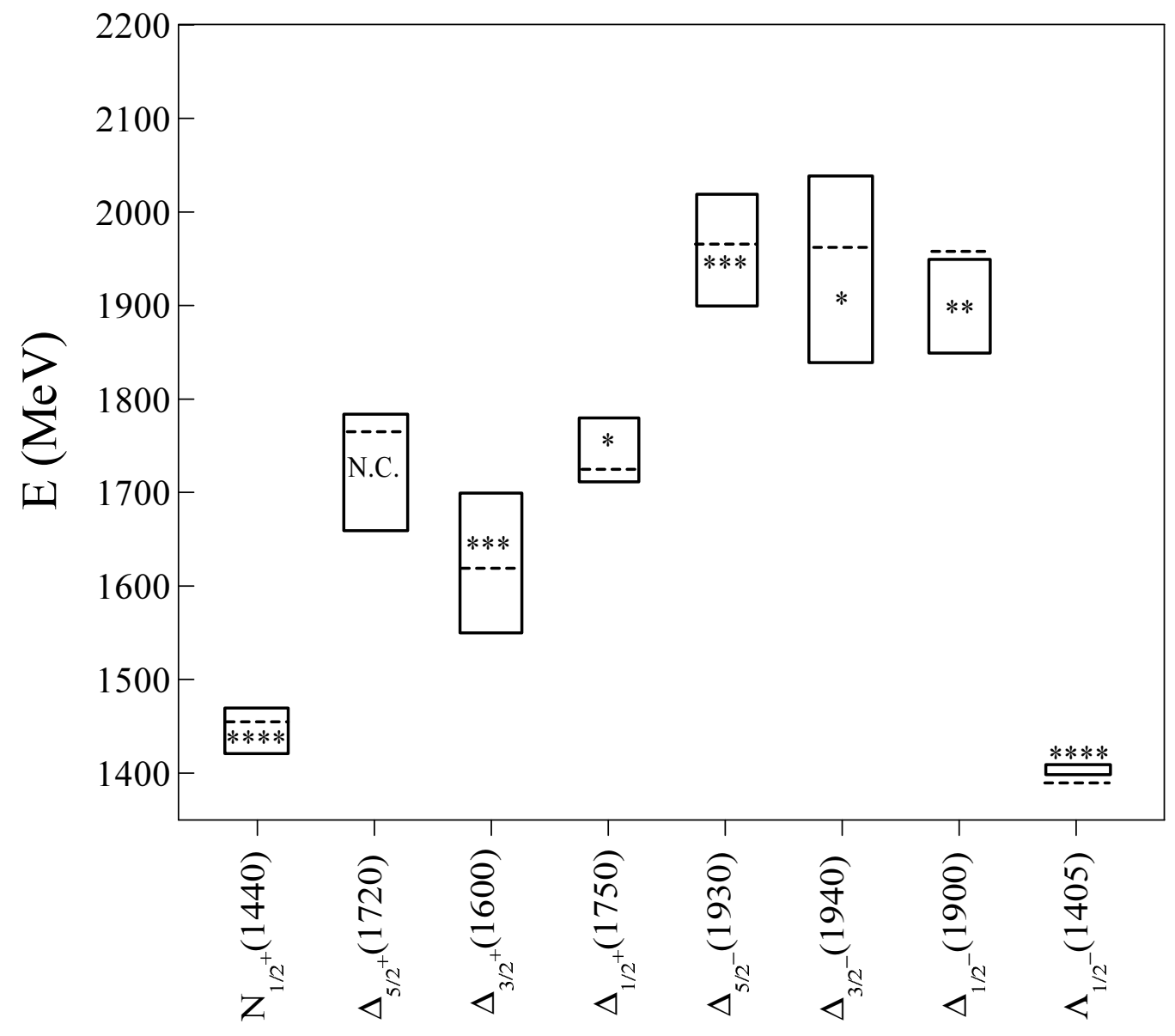

Figure 3 